\title{
Strain- and plasmid-level deconvolution of a synthetic metagenome by sequencing proximity ligation products
}

Metagenomics is a valuable tool for the study of microbial communities but has been limited by the difficulty of "binning" the resulting sequences into groups corresponding to the individual species and strains that constitute the community. Moreover, there are presently no methods to track the flow of mobile DNA elements such as plasmids through communities or to determine which of these are co-localized within the same cell. We address these limitations by applying $\mathrm{Hi}-\mathrm{C}$, a technology originally designed for the study of threedimensional genome structure in eukaryotes, to measure the cellular co-localization of DNA sequences. We leveraged $\mathrm{Hi}-\mathrm{C}$ data generated from a simple synthetic metagenome sample to accurately cluster metagenome assembly contigs into groups that contain nearly complete genomes of each species. The Hi-C data also reliably associated plasmids with the chromosomes of their host and with each other. We further demonstrated that $\mathrm{Hi}-\mathrm{C}$ data provides a long-range signal of strain-specific genotypes, indicating such data may be useful for high-resolution genotyping of microbial populations. Our work demonstrates that $\mathrm{Hi}-\mathrm{C}$ sequencing data provide valuable information for metagenome analyses that are not currently obtainable by other methods. This metagenomic $\mathrm{Hi}-\mathrm{C}$ method could facilitate future studies of the fine-scale population structure of microbes, as well as studies of how antibiotic resistance plasmids (or other genetic elements) mobilize in microbial communities. The method is not limited to microbiology; the genetic architecture of other heterogeneous populations of cells could also be studied with this technique. 
1 Christopher W. Beitel [1, 2], Lutz Froenicke [1], Jenna M. Lang [1], Ian F. Korf [1, 2], Richard

2 W. Michelmore [1, 2, 3], Jonathan A. Eisen [1, 4, 5], and Aaron E. Darling [6]

3 1. The University of California, Davis Genome Center, Davis, California, United States of

4 America

5 2. Department of Molecular \& Cellular Biology, University of California, Davis, California,

6 United States of America

7 3. Department of Plant Sciences, University of California, Davis, California, United States of

8 America

9 4. Department of Medical Microbiology and Immunology, University of California Davis,

10 California, United States of America

11 5. Department of Evolution and Ecology, University of California Davis, Davis, California,

12 United States of America

13 6. ithree institute, University of Technology Sydney, Sydney, NSW, Australia

14 Corresponding Authors: Christopher W. Beitel (ㅎ@ ucdavis.edu), Aaron E. Darling

15 (aaron.darling@uts.edu.au) 
17 Microbial ecology is the study of microbial communities in terms of their composition, functional 18 diversity, interactions, stability, and emergent properties (Handelsman, 2004; Konopka, 2009). Knowledge of the roles microbes play in ecosystems is essential for understanding how these ecosystems function (Konopka, 2009). Readily-cultivated organisms are estimated to constitute less than $1 \%$ of all microbial species, leading to the development of culture-independent methods for studying microbial communities (Gilbert \& Dupont, 2011; Hugenholtz, 2002; Staley, 1985). These culture-independent methods allow communities to be characterized directly.

Current sequencing-based metagenomic methods do not capture some of the most informative genetic information in microbial communities, in particular the long-range sequence contiguity and associations of genetic material in individual cells. In nearly all metagenomic methods, cells from the microbial community are lysed en masse to obtain a bulk DNA sample. This results in DNA from many different cells being mixed together, so that the genotype and species identity of individual cells are lost. Chromosomal DNA is then fragmented into pieces ( $~ 500$ bp to $40 \mathrm{kbp}$, depending on the sequencing strategy), further reducing contiguity.

Improved sample-processing workflows might preserve this information and thereby yield greater insight into the genetic structure of microbial communities. High throughput single-cell genomics (e.g. applied to thousands of cells) offers a promising alternative to shotgun metagenomics that preserves information about cellular compartmentalization of genetic material. These approaches are exquisitely sensitive to contamination by foreign DNA (from the sample itself, the laboratory environment, and from "ultra-pure" commercial reagents), necessitating the use of specialized equipment and reagents (Blainey, 2013; Woyke et al., 2011). Long-read technologies, such as Pacific Biosciences (Eid et al., 2009) and nanopore (Maitra, Kim, \& Dunbar, 2012) sequencing, may help address this challenge but are still constrained by the difficulty of preparing adequate amounts of very long DNA fragments.

Computational methods have been developed to infer genomic contiguity from metagenomic data by binning metagenome assembly contigs by species. These binning procedures pose a significant analytical challenge. Several methods have been developed that can be divided into comparative, compositional, and assembly approaches. Comparative approaches use alignments to reference sequences to assign contigs to species within existing taxonomies (Droge \& Mchardy, 2012). Comparative approaches are limited by their reliance on existing taxonomies. Compositional approaches form clusters of contigs that share similar oligomer (usually $4 \mathrm{bp}$ to $8 \mathrm{bp}$ ) composition (Droge \& Mchardy, 2012). Compositional approaches tend to be limited as well due to their underlying assumption that contigs with similar sequence composition belong together. Horizontal gene transfer complicates both of these analysis methods because it can introduce gene content from a taxonomically distant relative with unusual nucleotide composition. Thirdly, metagenome assembly can be viewed as a metagenome binning approach since sequences placed on the same scaffold are necessarily present in the same bin for any downstream binning procedure. As with all genome assembly approaches, metagenome assembly seeks to infer sequence adjacencies from paired- and long-read technologies. This 
approach is limited by the availability of such data that span large repetitive regions (Treangen et al., 2012; Howe et al., 2014; Treangen et al., 2013).

Binning seeks to address the challenge of determining which sequences were present within cells of the same species prior to DNA extraction. We reasoned that such co-localizations could be inferred from Hi-C data, a method originally developed for the study of threedimensional genome structure in eukaryotes (Lieberman-Aiden et al., 2009). This method relies on cross-linking molecules in close physical proximity and consequently identifies both intraand inter-chromosomal associations, reflecting the spatial arrangement of DNA at the time of cross-linking within intact nuclei or non-nucleated cells (Umbarger et al., 2011). We predicted that sequences of DNA not present in the same cell at the time of cross-linking would not be cross-linked together and should not be associated by Hi-C reads (Fig. S1). Herein we demonstrate the utility of $\mathrm{Hi}-\mathrm{C}$ as a tool for addressing metagenomic binning and related problems in microbial ecology. To do so we first constructed a synthetic microbial community by culturing and mixing five organisms with available reference genomes. We then performed a metagenome assembly on sequences that were simulated in silico from the genomes of these organisms. Our first objective was to group these metagenome assembly contigs according to species using $\mathrm{Hi}-\mathrm{C}$ reads that were generated from the synthetic microbial community. We then sought to differentiate two closely related E. coli strains included within this mixture. To do so we constructed contig and variant graphs and analyzed those graphs to characterize the extent to which Hi-C data might resolve the genotypes of species and strains present in our synthetic community.

\section{MATERIALS AND METHODS}

\section{Construction of a synthetic microbial community}

Pediococcus pentosaceus and Lactobacillus brevis were provided by the UC Davis Enology Culture Collection (http://wineserver.ucdavis.edu). Single colonies were used to start cultures in 5 $\mathrm{ml}$ liquid MRS broth. Escherichia coli BL21 (ATCC\# PTA-5073), E. coli K12 DH10B (ATCC\# 207214), and Burkholderia thailandensis (ATCC\# 700388) were obtained as freeze-dried stocks from the American Type Culture Collection (ATCC). The E. coli strains were re-suspended in 5 $\mathrm{ml}$ of LB liquid medium (10g/L Tryptone, $10 \mathrm{~g} / \mathrm{L} \mathrm{NaCl}, 5 \mathrm{~g} / \mathrm{L}$ Yeast Extract) and the $B$. thailandensis was re-suspended in $5 \mathrm{ml}$ of Nutrient Broth (Peptone $15.0 \mathrm{~g} / \mathrm{L}$, yeast extract $3 \mathrm{~g} / \mathrm{L}$, sodium chloride $6 \mathrm{~g} / \mathrm{L}, \mathrm{D}(+)$ glucose $1 \mathrm{~g} / \mathrm{L})$. All were incubated, with shaking, overnight at $37^{\circ} \mathrm{C}$ to produce starter cultures.

A separate $50 \mathrm{ml}$ culture for each organism was created by inoculation with $10 \mathrm{ul}$ of the appropriate starter culture and grown, with shaking, at $37^{\circ} \mathrm{C}$, for 24 hours. The cell density of each culture was estimated by measuring the OD600. The cultures were then mixed in quantities proportional to their optical density, seeking to have equal representation of each organism in the synthetic community. Glycerol was added to a final concentration of $7 \%$ and the final mixture was divided into $2 \mathrm{ml}$ tubes and frozen at $-80^{\circ} \mathrm{C}$. 
95

96

97

98

99

100

101

102

103

104

105

106

107

108

109

110

111

112

\section{3}

114

115

116

117

118

119

120

121

122

123

124

125

126

127

128

129

130

131

132

We simulated Illumina paired-end sequencing of the synthetic microbial community to obtain metagenomic assemblies that we could subsequently attempt to bin using experimentally derived Hi-C reads. Reads were simulated using Grinder (Angly, Willner, Rohwer, Hugenholtz, \& Tyson, 2012) v0.4.5, a tool for simulating metagenomic shotgun sequence reads. Replicons were sampled assuming uniform abundance of species. A total of 61,063,000 million reads were simulated to cover the genomes at 500x. From this set, paired-end read datasets of varying levels of coverage (100, 50, and 5x) were formed. Read length was simulated to $165 \mathrm{bp}$ and fragment size was simulated with a normal distribution around $550 \mathrm{bp}$ with a standard deviation of $50 \mathrm{bp}$ ("grinder -am uniform -cf \$cov -rd 165 -id 550 normal 50 -rf \$ref -fq 1 -q1 3010 -bn grinder.dp\$cov.\$tag”).

Assembly from each of the simulated metagenomic read sets was performed using SOAPdenovo (Luo et al., 2012) with a k-mer length of 23, yielding assemblies of varying quality (Table S1). Reads were aligned to the resulting assembly contigs using BWA MEM (Li, 2013). The rate of misassembly was determined by mapping contigs back to the reference assemblies (BWA-MEM with default parameters and a quality filter of MapQ>20) and counting the number of contigs that joined sequences from different species. These assemblies are available on Figshare: dx.doi.org/10.6084/m9.figshare.1004473, dx.doi.org/10.6084/m9.figshare.1004472, dx.doi.org/10.6084/m9.figshare.1004471.

\section{Application of Hi-C to the synthetic microbial community}

We performed Hi-C on the synthetic microbial community for the purpose of obtaining information that could be used to group (by species) the simulation-derived contigs described above, as well as to differentiate closely related strains present in our synthetic community. Hi-C was carried out by combining the cross-linking and cell wall digestion procedures described by (Umbarger et al., 2011) for bacterial 3-C experiments and the Hi-C protocol developed for mammalian cells (Lieberman-Aiden et al., 2009) with minor modifications. For additional details, see Supplementary Methods: Hi-C of Mixed Bacterial Cultures. Cells were transferred into a 50 $\mathrm{ml}$ centrifuge tube and washed three times in $25 \mathrm{ml}$ of TE buffer $(\mathrm{pH}=8.0)$ by centrifugation for 5 minutes at $4000 \mathrm{rpm}$ at $4^{\circ} \mathrm{C}$. Cells were re-suspended at an OD600 of 0.2 in TE and 37\% formaldehyde was added to a final concentration of $1 \%$ to cross-link proteins in the cell. Cells were incubated at room temperature for $30 \mathrm{~min}$ and subsequently for another $30 \mathrm{~min}$ on ice (Umbarger et al., 2011). The formaldehyde was quenched by adding glycine to a final concentration of $0.125 \mathrm{M}$ and incubated on ice for 10 minutes. After centrifugation, cells were resuspended in TE and lysozyme digestion was carried out as described to release the protein-DNA complexes (Umbarger et al., 2011). The samples were centrifuged and re-suspended in Hi-C lysis buffer and incubated on ice for 15 minutes (Lieberman-Aiden et al., 2009). From this step on the original Hi-C protocol employing HindIII (Lieberman-Aiden et al., 2009) was applied with some modifications. To summarize the Lieberman-Aiden et al. (2009) protocol, DNA in the crosslinked protein complexes is digested with HindIII endonuclease following cell lysis and free 
133 DNA ends are tagged with biotin. Blunt-ended DNA fragments are ligated under highly dilute

134 conditions, resulting in preferential ligation of fragments that are within the same cross-linked

135 DNA/protein complex. Next, crosslinks are removed, DNA is purified, biotin is eliminated from

136 un-ligated ends, DNA is size-selected, and ligation products are selected for through a biotin pull-

137 down. One modification we made to the published procedure was to reduce the concentration of

138 biotin-14-dCTP (Life Technologies) by half. Also, ligation to Illumina-compatible sequencing

139 adapters (Biooscientific) was carried out in solution before capture with streptavidin beads. The

140 DNA sample was size selected after end-repair and before adapter ligation by gel extraction for

141 fragment sizes ranging from 280 to $420 \mathrm{bp}$. The bead-captured Hi-C library was amplified by 10

142 cycles of PCR before a final cleanup with Ampure XP beads (Agencourt). The library was

143 sequenced in a single run on an Illumina Miseq machine using 160 bp paired-end reads.

\section{Sequence alignment and quality filtering}

145 Reference assembly sequences were obtained from the NCBI RefSeq database (Pruitt, Tatusova, 146 Brown, \& Maglott, 2012) with the following accession numbers for each of P. pentosaceus

147 (NC_008525), L. brevis (NC_008497, NC_008498, NC_008499), E. coli BL21 (NC_012892), E. 148 coli K12 DH10B (NC_010473), and B. thailandensis (NC_007651, NC_007650). These

149 sequences were pooled into a single reference database for sequence alignment. No quality

150 filtering was performed on raw reads, leaving this to be performed later using alignment quality

151 scores. Split-read sequence alignment was performed (independently for each read in a pair)

152 using BWA MEM (Li, 2013; default parameters) against the pooled reference assemblies as well

153 as (separately) against the metagenome assembly described above. Heat map visualizations and

154 insert distribution plots were generated from unfiltered alignments using custom R (The R Core

155 Development Team, 2010) scripts (see github.com/cb01/proxmine).

156 We investigated the effect of various alignment filtering parameters on the subsequent

157 variant graph analysis (illustration of the concept of a variant graph can be found in Fig. S7). To

158 this end, alignments of Hi-C reads to the reference genomes were filtered according to 24

159 parameter combinations, with three minimum mapping quality $(0,20,60)$, two CIGAR filtering

160 (none, $\mathrm{CIGAR}=160 \mathrm{M})$, and four minimum insert filtering $(0,1 \mathrm{~kb}, 10 \mathrm{~kb}, 40 \mathrm{~kb})$ conditions. In the

161 latter, Hi-C read pairs were excluded when their alignments within the reference assembly had an

162 insert size below the specified minimum, including cases of alignments spanning the linearization

163 points of these assemblies (e.g. for E. coli, near coordinates 0 and 4686137). An open-source

164 graph visualization tool, Gephi (0.8.2-beta), was used to visualize the Hi-C contig association

165 network (Bastian et al., 2009).

\section{Contig clustering}

167 We inferred grouping of metagenome assembly contigs by applying the Markov Clustering 168 Algorithm (MCL) to a matrix of contig association data (van Dongen, 2000). MCL is an

169 unsupervised clustering algorithm which simulates flow and accumulation of edge weights within

170 a given weighted graph structure. It has a computational complexity of $\mathrm{O}\left(\mathrm{Nk}^{2}\right)$ given an

171 implementation designed for sparse matrices. The matrix of edge weights provided to this 
172 algorithm was computed from contig association counts by normalizing edge weights according

173 to the following formula, which corrects for the expected inflation of association between large

174 contigs. Specified formally, let $L$ be a set of contig lengths with member $l_{i}$ denoting the length of

175 contig $i$. Given a contig pair $\{i, j\}$, let $c_{i j}$ denote the number of Hi-C read pairs with one end

176 aligning in contig $i$ and the other end in contig $j$. This count was normalized by the ratio of the

177 square of the maximum contig length and the lengths $l_{i}$ and $l_{j}$ of contigs $i$ and $j$, respectively.

$$
c_{i j}^{\prime}=\frac{\max (L)^{2} c_{i, j}}{l_{i} l_{j}}
$$

Prior to normalization, we filtered the contig association data for (1) contig associations greater than some minimum $\mathrm{k}$, and (2) associations between contigs of size greater than $\mathrm{L}$. We explored the $(\mathrm{k}, \mathrm{L})$ parameter space by performing normalization and MCL clustering for 205 parameter combinations, with 41 contig size minimums chosen evenly across [0,40000] and five contact minimums, $\{0,3,5,7,9\}$. For each of the 205 filtering parameter combinations, clustering was performed using 100 different MCL inflation values chosen to span the interval [1,2] in increments of 0.01 .

\section{Assessment of clustering quality}

Each metagenome assembly contig was aligned to the reference assemblies to determine its species or strain of origin, allowing us to determine which strains were present in each cluster of contigs. This was done by extracting every $70 \mathrm{bp}$ substring of the available contig sequences and aligning each of those back to the reference assemblies with BWA-MEM (default parameters, MapQ>20). A contig was designated as originating from the species to which the greatest number of these substrings aligned. A measure of clustering quality was computed by sampling random pairs of contigs ( $\mathrm{N}=100,000$ pairs) and comparing their cluster assignments to their species of origin. This random sampling was weighted according to the size of each contig such that the probability of sampling any contig from the set was equal to the size of that contig divided by the sum total of all contig sizes. Any contig not present in the clustering solution (but which was present as input for the clustering run that generated that solution) was added to the solution in a singleton cluster. For each sampled contig pair, if those contigs belonged to the same species and had been placed in the same cluster, they were counted as a true positive ("TP"). If they originated from the same species but had been placed in different clusters, they were counted as a false negative ("FN"). Likewise, contigs originating from different species that were placed in the same or different clusters were counted as false positives ("FP") and true negatives ("TN"), respectively. The true positive rate (a.k.a. sensitivity, recall), false positive rate, positive predictive value (a.k.a. precision), and negative predictive value were calculated from these counts according to standard formulae. All clustering quality measures were computed in two ways, one treating the two E. coli strains as independent classes (strain-level) and another treating them as the same (species-level).

\section{Analysis of SNP graph connectivity}


A SNP graph is an undirected graph wherein SNP sites are nodes and edges link pairs of SNP sites that were observed together in a sequence read pair. We expected SNP graphs that were constructed using Hi-C data to be more densely connected than SNP graphs that were constructed using mate-pair data because $\mathrm{Hi}-\mathrm{C}$ read pairs can span entire chromosomes while reads from mate-pair libraries span no more than $40 \mathrm{~kb}+/-5 \mathrm{~kb}$. In this way, Hi-C provides global information while that provided by mate pairs is locally constrained. To quantify this, SNP graphs were constructed for the Hi-C data and simulated mate-pair data and the shortest path between randomly chosen SNP pairs was plotted relative to the distance between the those variants within the reference assembly. These graphs were constructed from alignments of reads to the E. coli K12 reference assembly after it had been masked at variant positions identified through pairwise sequence alignment of E. coli K12 and BL21 using progressiveMauve (Darling, Mau, \& Perna, 2010). In this way, the masked $\mathrm{K} 12$ reference assembly was used as a scaffold for our analysis of Hi-C and mate-pair variant graphs.

Hi-C read datasets typically contain a mixture of reads derived from ligation and nonligation products, the latter having short inserts. In our analysis of SNP graph connectivity we sought to understand the contribution of these non-ligation products on the connectivity gains seen with Hi-C reads over mate-pairs. For comparison to our Hi-C reads, mate-pair read sets were computationally simulated for a range of sizes $(5 \mathrm{~kb}, 10 \mathrm{~kb}, 20 \mathrm{~kb}, 40 \mathrm{~kb})$. These were compared to three $\mathrm{Hi}-\mathrm{C}$ read sets: the entire $\mathrm{Hi}-\mathrm{C}$ dataset, $\mathrm{Hi}-\mathrm{C}$ reads with inserts below $1 \mathrm{~kb}$, and $\mathrm{Hi}-\mathrm{C}$ reads with inserts above $1 \mathrm{~kb}$.

SNP graph connectivity was analyzed using the simulated mate-pair read sets described above combined with Hi-C reads aligned and filtered for alignment qualities above or equal to 60 and for CIGAR encodings of 160M Read pairs with both ends aligning to SNP positions were identified and for each corresponding SNP pair an edge was formed. Shortest path lengths between sampled SNP positions were computed using a custom breadth-first search program, relying on the Boost Graph Library (http://boost.org). The program constructs a graph from a user-specified SNP edge list and performs a breadth-first search to identify the shortest path length between a user-specified number of randomly selected SNP pairs. We calculated path lengths between 10,000 randomly chosen SNP pairs. To aid in visualization, the full range of variant separation distances was divided into $20 \mathrm{~kb}$ segments and the average path length was computed for each segment. These data were smoothed using locally-weighted scatterplot smoothing (LOWESS).

\section{RESULTS}

\section{The synthetic microbial community for metagenomic Hi-C}

242 Five microorganisms were chosen to test the metagenomic Hi-C approach: Lactobacillus brevis, 243 Pediococcus pentosaceus, Burkholderia thailandensis, Escherichia coli K12 DH10B and E. coli 244 BL21 (DE3). These were selected because high quality reference genomes are available. In 245 addition, the multiple replicons of $B$. thailandensis and plasmids present in L. brevis allowed us 
246 to explore whether Hi-C might link separate replicons present in the same cell. We selected two 247 strains of E. coli (K12 and BL21) to evaluate whether Hi-C sequence data could be used to

248 resolve inter-strain differences. Genome alignment of these two E. coli shows that $87.9 \%$ of their 249 genomes can be aligned and that the average nucleotide identity across aligned regions is $99.5 \%$. 250 Finally, because differences in cell membrane structure and GC content could potentially lead to 251 bias in DNA extraction and/or cross-linking efficiency, we selected two lactic acid bacteria $(P$.

252 pentosaceus and L. brevis), which are low-GC, Gram-positive organisms for which only $39.5 \%$ 253 of their genomes can be aligned with $84.3 \%$ average nucleotide identity across aligned regions.

\section{Metagenome assembly}

255 We generated a metagenome assembly of the synthetic microbial community that we could use as 256 input for our analysis of the utility of $\mathrm{Hi}-\mathrm{C}$ for species clustering. Hi-C sequencing data is biased 257 by the distribution of restriction sites for the restriction enzyme used to construct the library as 258 well as by other factors including GC content, restriction fragment length, and "mappability" 259 (Yaffe \& Tanay, 2011). Hi-C data contain numerous chimeric sequences and thus are not suitable 260 for de novo contig assembly. Therefore, we simulated and assembled Illumina metagenomic 261 sequence data at varying coverage levels to yield the assemblies summarized in (Table S1). The 262 size (bp) of each of these assemblies was approximately 77\% of the sum of the synthetic 263 community reference genome sizes and this fraction did not change when increasing the quantity 264 of input reads from 5x to 100x. Alignment of assembled contigs to the collection of reference 265 genomes indicates that similar regions of the two $E$. coli genomes were co-assembled into single contigs (data not shown). The assembly on the lowest amount of input sequence (5x coverage) contained two misassembled contigs and three misassembled scaffolds. Assemblies at 50x and 100x coverage were free from misassembled contigs and scaffolds and were similar in terms of their contig counts, N50s, and total amounts of sequence assembled. We used the 100x coverage assembly (SOAP-3) for all further analysis.

\section{Hi-C Library Statistics}

A total of 20,623,187 read pairs were obtained from a single MiSeq run to yield $\sim 6.4 \mathrm{~Gb}$ of raw sequence data. Of these, $98.25 \%$ could be aligned back to the reference genomes by BWA MEM. A total of $21,260,753$ (51.55\% of original and $52.46 \%$ of raw aligned reads) read pairs were retained after filtering for both reads in the pair aligning at high quality (MapQ $>=60)$ and in full $(\mathrm{CIGAR}=160 \mathrm{M})$. Due to either self-ligation or imperfect enrichment for ligation junctions, most of the reads present in the dataset represent local genomic DNA fragments. Therefore we classify read pairs mapping within 1,000 nt as fragment reads, while all other reads are considered to be $\mathrm{Hi}-\mathrm{C}$ reads ( $3 \%$ of reads). The abundance of each replicon was estimated using filtered alignments and unfiltered alignments (Table 1), as well as by normalizing each unfiltered alignment count with the restriction site counts for each replicon (Table S2). These figures can only be used as approximate measures of abundance because these values are affected by the frequency of restriction sites in each organism and a multitude of other confounding factors 
284 (Morgan, Darling, \& Eisen, 2010). Insert distances derived from the alignment of Hi-C reads to

285 the E. coli K12 genome were distributed in a similar manner as previously reported (Fig. 1;

286 Lieberman-Aiden et al., 2009). We observed a minor depletion of alignments spanning the

287 linearization point of the E. coli K12 assembly (e.g. near coordinates 0 and 4686137) due to edge

288 effects induced by BWA treating the sequence as a linear chromosome rather than circular.

\section{Clustering contigs by species with Hi-C}

290 The experimentally derived Hi-C read pairs have a long tail in their insert distribution (Fig. 1;

291 Figs. S3-S6) indicating that they provide information that can be used to link metagenome

292 assembly contigs originating from distant parts of the same chromosome. We evaluated whether

293 Hi-C reads could be used to group the simulated assembly contigs described above into clusters

294 that correspond with each species' genome. We tested this process on the SOAP-3 assembly,

295 using only contigs with a length of at least $5 \mathrm{kbp}$. This threshold was applied to exclude short

296 contigs that may not have a HindIII restriction site. As HindIII recognizes a 6bp motif, it cuts on

297 average every 4,096bp. We note that $>25 \%$ of a $4 \mathrm{Mbp}$ genome is expected to have inter-site

298 distances $>10 \mathrm{Kbp}$ in simulations that treat 6-cutter restriction sites as uniformly distributed (data

299 not shown) suggesting that many of the contigs < $10 \mathrm{Kbp}$ may cluster poorly due to lack of

300 restriction sites. The dataset was further reduced to exclude links among contig pairs which are

301 associated by 5 or fewer read pairs.

302 We tested Markov clustering (van Dongen, 2000) on these data over a range of inflation

303 (affecting cluster solution granularity) parameters (Table 2). In the best case, Markov clustering

304 produced four clusters, each of which correspond to the nearly complete genome of a species in

305 our synthetic community. In this clustering, the two strains of E. coli appear in the same group.

306 When using the default inflation parameter of 2.0 we find that the data is under-clustered, but

307 there are no false positive associations among contigs for this choice of input.

308 To further understand the sensitivity of MCL to choices of filtering and inflation

309 parameters, we performed clustering across the 204 filtering and 100 inflation (total of 20,400)

310 conditions (see Methods: Contig Clustering) using MCL. A representative subset of all parameter

311 combinations tested is shown in Fig. S2. These data suggest that once a sufficient contact and

312 contig size minimums have been applied, cluster solutions vary primarily in terms of their

313 granularity (as the inflation parameter varies), not their PPV (remaining close to 1) or FPR

314 (remaining close to 0 ). Low inflation values, close to 1, give clustering solutions with the highest

315 TPR's, but this does not hold true without sufficient filtering.

\section{Association of species with metagenomic $\mathrm{Hi}-\mathrm{C}$ data}

317 We next sought to quantify the cellular co-localization signal underlying the above-described

318 species clustering. For this analysis we studied Hi-C reads aligned directly to the reference

319 assemblies of the members of our synthetic microbial community with the same alignment

320 parameters as were used in the top ranked clustering (described above). We first counted the

321 number of Hi-C reads associating each reference assembly replicon (Fig. 2; Table S3), observing 
322 that Hi-C data associated replicons within the same species (cell) orders of magnitude more

323 frequently than it associated replicons from different species. The rate of within-species

324 association was $98.8 \%$ when ignoring read pairs mapping less than 1,000 bp apart. Including read

325 pairs $<1,000 \mathrm{bp}$ inflated this figure to $99.97 \%$. Figure 3 illustrates this by visualizing the graph of

326 contigs and their associations. Similarly, for the two E. coli strains (K12, BL21) we observed the

327 rate of within-strain association to be $96.36 \%$. When evaluated on genes unique to each strain

328 (where read mapping to each strain would be unambiguous), the self-association rate was

329 observed to be $>99 \%$.

330 We observed that the rate of association of L. brevis plasmids 1 and 2 with each other and

331 with the L. brevis chromosome was at least 100-fold higher than with the other constituents of the

332 synthetic community (Fig. 2). Chromosome and plasmid Hi-C contact maps show that the

333 plasmids associate with sequences throughout the L. brevis chromosome (Fig. 4; Figs. S3-S5) and

334 exhibit the expected enrichment near restriction sites. This demonstrates that metagenomic Hi-C

335 can be used to associate plasmids to specific strains in microbial communities as well as to

336 determine cell co-localization of plasmids with one another.

\section{Variant graph connectedness}

338 Algorithms that reconstruct single-molecule genotypes from samples containing two or more 339 closely-related strains or chromosomal haplotypes depend on reads or read pairs that indicate 340 whether pairs of variants coexist in the same DNA molecule. Such algorithms typically represent

341 the reads and variant sites as a variant graph wherein variant sites are represented as nodes, and 342 sequence reads define edges between variant sites observed in the same read (or read pair). We 343 reasoned that variant graphs constructed from $\mathrm{Hi}-\mathrm{C}$ data would have much greater connectivity

344 (where connectivity is defined as the mean path length between randomly sampled variant 345 positions) than graphs constructed from mate-pair sequencing data, simply because Hi-C inserts

346 span megabase distances. Such connectivity should, in theory, enable more accurate 347 reconstruction of single-molecule genotypes from smaller amounts of data. Furthermore, by

348 linking distant sites with fewer intermediate nodes in the graph, estimates of linkage disequilibrium at distant sites (from a mixed population) are likely to have greater precision.

To evaluate whether Hi-C produces more connected variant graphs we compared the 351 connectivity of variant graphs constructed from $\mathrm{Hi}-\mathrm{C}$ data to those constructed from simulated 352 mate-pair data (with average inserts of $5 \mathrm{~kb}, 10 \mathrm{~kb}, 20 \mathrm{~kb}$, and $40 \mathrm{~kb}$ ). To exclude paired-end 353 products from the analysis, Hi-C reads with inserts under $1 \mathrm{~kb}$ were excluded from the analysis.

354 For each variant graph constructed from these inputs, 10,000 variant position pairs were sampled 355 at random, with $94.75 \%$ and $100 \%$ of these pairs belonging to the same connected graph

356 component of the $\mathrm{Hi}-\mathrm{C}$ and $40 \mathrm{~kb}$ variant graphs, respectively. These rates fell to $6.21 \%, 16.6 \%$, 357 and $32.38 \%$ for the $5 \mathrm{~kb}, 10 \mathrm{~kb}$, and $20 \mathrm{~kb}$ mate-pair variant graphs, respectively (Table 3 ).

358 Across conditions, variant graphs differed in terms of their connectivity, with Hi-C graphs

359 showing the greatest connectivity. Despite having simulated an equal number of reads for each

360 mate-pair distance, the numbers of variant positions linked by such reads was different across

361 conditions. We observed that the variant graph derived from $\mathrm{Hi}-\mathrm{C}$ data $(>1 \mathrm{~kb}$ inserts, no 
362 alignment filtering), despite having the lowest number of variant links, had the lowest mean and

363 maximum path length $(5.47,11$; Table 3$)$. Path length was not correlated with distance within Hi-

$364 \mathrm{C}$ variant graphs, in contrast to the mate-pair conditions (Fig. 5). The lengths of paths between

365 variant pairs in the mate-pair graphs did increase with distance, reaching maximums of 71, 96,

36694 , and 111 in the $5 \mathrm{~kb}, 10 \mathrm{~kb}, 20 \mathrm{~kb}$, and $40 \mathrm{~kb}$ cases, respectively. We further examined the

367 effect of alignment quality and completeness filtering and observed that in the latter case such

368 filtering vastly reduced the rate at which variant positions occur within the same connected graph

369 component.

370

371

372

373

374

375

376

377

378

379

380

381

382

383

384

385

386

387

388

389

390

391

392

393

394

395

396

397

398

399

400

401

\section{DISCUSSION}

This study demonstrates that Hi-C sequencing data provide valuable information for metagenome analyses that are not currently obtainable by other methods. By applying Hi-C to a synthetic microbial community we showed that genomic DNA was associated by Hi-C read pairs within strains orders of magnitude more frequently than between strains. Hi-C reads associated genomic regions at distances not achievable with mate-pair or long-read sequencing technologies. The long-range contiguity information provided by $\mathrm{Hi}-\mathrm{C}$ reads enabled us to perform species-level clustering of metagenome assembly contigs with perfect precision and recall scores when the input had been filtered sufficiently. We performed an exploration of the clustering parameter space to understand the factors affecting clustering quality and identified a number of key filtering parameters. Optimal filtering involved retention of only contigs that are large enough to contain (or occur near) a HindIII restriction site and furthermore to remove low-frequency contig associations that constitute a form of "noise". Additional work is needed to develop methods to identify and remove "noise" from Hi-C datasets. Lastly, we compared the connectivity of variant graphs constructed from mate-pair and $\mathrm{Hi}-\mathrm{C}$ read datasets, observing much greater connectivity in the latter case, illustrating the global nature of the Hi-C signal.

We also observed orders of magnitude greater rates of association between plasmids and chromosomes of their hosts than between plasmids and the genomes of other species. Based on this observation, we believe Hi-C has the potential to be used to study horizontal gene transfer. Given a metagenome assembly, Hi-C provides a means to link plasmid sequences to chromosomes of the host strain, and may provide the means to detect cases where plasmids have been transferred among co-existing species of bacteria. We have thus far demonstrated that Hi-C provides a signal of cell co-localization for the two plasmids present within the L. brevis genome. Alternative methods do not allow identification of which cells in a microbial community harbor such mobile DNA elements. Hi-C data has the potential to help quantify the dynamics of horizontal gene transfer and help characterize the spread of antibiotic resistance and virulence factors. It remains to be determined whether this signal will be sufficient to localize small, lowcopy, or highly variable mobile elements within the species that contain them.

The resolving power of Hi-C and related methods such as ChIA-PET (Fullwood et al., 2009) when applied to complex natural microbial communities is as of yet undetermined. In principle, as the number of species and genotypes in a community grows the amount of sequence data required to resolve species and strains also grows. This challenge is common to all 
metagenomic approaches and is not specific to the $\mathrm{Hi}-\mathrm{C}$ method described. Improvements on metagenomic analysis of complex communities may require integration of $\mathrm{Hi}-\mathrm{C}$ data with other information sources such as sequence composition, phylogeny, and measurements of abundance.

The problems of differentiating contigs originating from different species is similar to that of differentiating contigs originating from different chromosomes of the same species. Recently a study reported the use of Hi-C to perform genome scaffolding of several individual eukaryotic genomes, first by inferring chromosomal groupings of contigs and then ordering sequences along the chromosome (Burton et al., 2013). Markov Clustering of Hi-C association data may be used to cluster contigs into chromosomal groups without specifying the number of chromosomes $a$ priori. This may be important for samples where the number of chromosomes (e.g. tumor samples), species, or species abundances (e.g. environmentally-isolated microbial communities) are not known.

Hi-C analysis can be applied to communities other than environmentally-isolated microbial communities, such as pools of BAC clones. Heterogeneous tumor populations are analogous in some ways to microbial communities and $\mathrm{Hi}-\mathrm{C}$ may be applied to identify subpopulations therein. The problem of resolving the membership of variants in closely related strains (between different cells) shares some common features with the problem of differentiating closely related haplotypes within polyploid eukaryotic genomes (within the same cells). Recent work has demonstrated that Hi-C data can be used to phase haplotypes in a diploid organism (Selvaraj, R Dixon, Bansal, \& Ren, 2013). Our analysis indicates that the average degree of separation between variants within a Hi-C variant graph is dramatically lower than that in matepair variant graphs. This is significant because as the degree of separation between distant graph regions grows, error is compounded and the reliability of inferences regarding the phase of these regions declines. Our analysis thus indicates that $\mathrm{Hi}-\mathrm{C}$ data provide an informative signal for the analysis of haplotype and strain mixtures.

\section{AVAILABILITY}

Raw sequence data for this experiment has been deposited to the NCBI Short Read Archive, accession SRX377733. Analysis scripts can be found at github.com/cb01/proxmine. Simulated assemblies are available at dx.doi.org/10.6084/m9.figshare.1004473, dx.doi.org/10.6084/m9.figshare.1004472, and dx.doi.org/10.6084/m9.figshare.1004471.

\section{AUTHOR CONTRIBUTIONS}

Prepared the mixture: J.M.L. Prepared Hi-C Libraries: L.F. and C.B. Analyzed the data C.B. and A.D. Wrote the paper: All. Conceived the method: C.B. and A.D. Designed the experiment: All.

\section{ACKNOWLEDGEMENTS}

We wish to acknowledge the substantial efforts of Michael Lewis, administrator of the UC Davis Genome Center Cluster Computing Resource, to provide and maintain the computing resources on which these analyses were performed. We would also like to acknowledge Matthew DeMaere (UTS) for his contribution to Fig. 3. 
This work was supported by a gift from MARS, Inc. and by Department of Homeland Security contract \#HSHQDC-11-C-00091.

443

444

445

446

447

448

449

450

451

452

453

454

455

456

457

458

459

460

461

462

463

464

465

466

467

468

469

470

471

472

473

474

475

476

477

478

479

480

481

482

483

484

\section{REFERENCES}

Angly, F. E., Willner, D., Rohwer, F., Hugenholtz, P., \& Tyson, G. W. (2012). Grinder: a versatile amplicon and shotgun sequence simulator. Nucleic acids research, 40(12), e94. doi:10.1093/nar/gks251

Avis, D. (1978). Two Greedy Heuristics for the Weighted Matching Problem. Proceedings of the 9th S-E Conference on Combinatorics, Graph Theory, and Computing (pp. 55-76).

Bastian, M., Heymann, S. \& Jacomy, M. (2009). Gephi: An Open Source Software for Exploring and Manipulating Networks.. In E. Adar, M. Hurst, T. Finin, N. S. Glance, N. Nicolov \& B. L. Tseng (eds.), The International Conference on Weblogs and Social Media, : The AAAI Press. ISBN: 978-1-57735-421-5

Blainey, P. (2013). The future is now: single-cell genomics of bacteria and archaea. FEMS microbiology reviews, 37(3), 1-29. doi:10.1111/1574-6976.12015.

Burton, J. N., Adey, A., Patwardhan, R. P., Qiu, R., Kitzman, J. O., \& Shendure, J. (2013). Chromosome-scale scaffolding of de novo genome assemblies based on chromatin interactions. Nature biotechnology, (November). doi:10.1038/nbt.2727

Darling, A. E., Mau, B., \& Perna, N. T. (2010). progressiveMauve: multiple genome alignment with gene gain, loss and rearrangement. PloS one, 5(6), e11147. doi:10.1371/journal.pone.0011147

Droge, J., \& Mchardy, A. C. (2012). Taxonomic binning of metagenome samples generated by next-generation sequencing technologies, 13(6), 646-655. doi:10.1093/bib/bbs031

J. Eid, A. Fehr, J. Gray, K. Luong, J. Lyle, G. Otto, P. Peluso, D. Rank, P. Baybayan, B. Bettman, A. Bibillo, K. Bjornson, B. Chaudhuri, F. Christians, R. Cicero, S. Clark, R. Dalal, J. Dixon, M. Foquet, A. Gaertner, P. Hardenbol, C. Heiner, K. Hester, D. Holden, G. Kearns, X. Kong, R. Kuse, Y. Lacroix, S. Lin, P. Lundquist, C. Ma, P. Marks, M. Maxham, D. Murphy, I. Park, T. Pham, M. Phillips, J. Roy, R. Sebra, G. Shen, J. Sorenson, A. Tomaney, K. Travers, M. Trulson, J. Vieceli, J. Wegener, D. Wu, A. Yang, D. Zaccarin, P. Zhao, F. Zhong, J. Korlach, and S. Turner, "Real-Time DNA Sequencing from Single Polymerase Molecules," no. January, pp. 133-138, 2009.

Ellson, J., Gansner, E. R., Koutsofios, E., North, S. C., \& Woodhull, G. (2003). Graphviz and Dynagraph - Static and Dynamic Graph Drawing Tools.

M. J. Fullwood, M. H. Liu, Y. F. Pan, J. Liu, H. Xu, Y. Bin Mohamed, Y. L. Orlov, S. Velkov, A. Ho, P. H. Mei, E. G. Y. Chew, P. Y. H. Huang, W.-J. Welboren, Y. Han, H. S. Ooi, P. N. Ariyaratne, V. B. Vega, Y. Luo, P. Y. Tan, P. Y. Choy, K. D. S. A. Wansa, B. Zhao, K. S. Lim, S. C. Leow, J. S. Yow, R. Joseph, H. Li, K. V Desai, J. S. Thomsen, Y. K. Lee, R. K. M. Karuturi, T. Herve, G. Bourque, H. G. Stunnenberg, X. Ruan, V. Cacheux-Rataboul, W.-K. Sung, E. T. Liu, C.-L. Wei, E. Cheung, and Y. Ruan, "An oestrogen-receptoralpha-bound human chromatin interactome.," Nature, vol. 462, no. 7269, pp. 58-64, Nov. 2009.

Gilbert, J. A., \& Dupont, C. L. (2011). Microbial Metagenomics: Beyond the Genome. Annual Review of Marine Science, 3(1), 347-371. doi:10.1146/annurev-marine-120709-142811

Handelsman, J. (2004). Metagenomics : Application of Genomics to Uncultured Microorganisms Metagenomics : Application of Genomics to Uncultured Microorganisms, 68(4). doi:10.1128/MBR.68.4.669

Howe, A. C., Jansson, J. K., Malfatti, S. A., Tringe, S. G., Tiedje, J. M., \& Brown, C. T. (2014). Tackling soil diversity with the assembly of large, complex metagenomes. Proceedings of 
the National Academy of Sciences of the United States of America, 111(13), 4904-9. doi:10.1073/pnas.1402564111

Hugenholtz, P. (2002). Exploring prokaryotic diversity in the genomic era. Genome biology, 3(2), REVIEWS0003.

Konopka, A. (2009). What is microbial community ecology? The ISME journal, 3(11), 1223-30. doi:10.1038/ismej.2009.88

Li, H. (2013). Aligning sequence reads, clone sequences and assembly contigs with BWA-MEM, Oxford University Press (arxiv.org), 00(00), 1-3.

E. Lieberman-Aiden, N. L. van Berkum, L. Williams, M. Imakaev, T. Ragoczy, A. Telling, I. Amit, B. R. Lajoie, P. J. Sabo, M. O. Dorschner, R. Sandstrom, B. Bernstein, M. a Bender, M. Groudine, A. Gnirke, J. Stamatoyannopoulos, L. a Mirny, E. S. Lander, and J. Dekker, "Comprehensive mapping of long-range interactions reveals folding principles of the human genome.," Science (New York, N.Y.), vol. 326, no. 5950, pp. 289-93, Oct. 2009.

R. Luo, B. Liu, Y. Xie, Z. Li, W. Huang, J. Yuan, G. He, Y. Chen, Q. Pan, Y. Liu, J. Tang, G. Wu, H. Zhang, Y. Shi, Y. Liu, C. Yu, B. Wang, Y. Lu, C. Han, D. W. Cheung, S.-M. Yiu, S. Peng, Z. Xiaoqian, G. Liu, X. Liao, Y. Li, H. Yang, J. Wang, T.-W. Lam, and J. Wang, "SOAPdenovo2: an empirically improved memory-efficient short-read de novo assembler.," GigaScience, vol. 1, no. 1, p. 18, Jan. 2012.

Maitra, R. D., Kim, J., \& Dunbar, W. B. (2012). Recent advances in nanopore sequencing. Electrophoresis, 33(23), 3418-28. doi:10.1002/elps.201200272

Morgan, J. L., Darling, A. E., \& Eisen, J. A. (2010). Metagenomic sequencing of an in vitrosimulated microbial community. PloS one, 5(4), e10209. doi:10.1371/journal.pone.0010209

Pruitt, K. D., Tatusova, T., Brown, G. R., \& Maglott, D. R. (2012). NCBI Reference Sequences (RefSeq): current status, new features and genome annotation policy. Nucleic acids research, 40(Database issue), D130-5. doi:10.1093/nar/gkr1079

Selvaraj, S., Dixon, J. R., Bansal, V., \& Ren, B. (2013). Whole-genome haplotype reconstruction using proximity-ligation and shotgun sequencing. Nature biotechnology, (November). doi:10.1038/nbt.2728

Staley, J. T. (1985). Microorganisms in aquatic and terrestrial habitats. Annual Review of Microbiology. 10.1146/annurev.mi.39.100185.001541

Treangen, T. J., \& Salzberg, S. L. (2012). Repetitive DNA and next-generation sequencing: computational challenges and solutions. Nature reviews. Genetics, 13(1), 36-46. doi:10.1038/nrg3117

Treangen, T. J., Koren, S., Sommer, D. D., Liu, B., Astrovskaya, I., Ondov, B., Darling, A. E., Phillippy, A. M., Pop, M. (2013). MetAMOS: a modular and open source metagenomic assembly and analysis pipeline. Genome biology, 14(1), R2. doi:10.1186/gb-2013-14-1-r2

van Dongen, S. (2000), Graph Clustering by Flow Simulation. PhD thesis, University of Utrecht (May).

The R Core Development Team. (2010). $R$ : A Language and Environment for Statistical Computing (Vol. 1). Vienna, Austria.: R Foundation for Statistical Computing. Retrieved from http://www.r-project.org/

M. Umbarger, E. Toro, M. Wright, G. J. Porreca, D. Baù, S.-H. Hong, M. J. Fero, L. J. Zhu, M. a Marti-Renom, H. H. McAdams, L. Shapiro, J. Dekker, and G. M. Church, "The three-dimensional architecture of a bacterial genome and its alteration by genetic perturbation.," Molecular cell, vol. 44, no. 2, pp. 252-64, Oct. 2011. 
528 Woyke, T., Sczyrba, A., Lee, J., Rinke, C., Tighe, D., Clingenpeel, S., Malmstrom, R.,

529 Stepanauskas, R., \& Cheng, J. (2011). Decontamination of MDA reagents for single cell

$530 \quad$ whole genome amplification. PloS one, 6(10), e26161. doi:10.1371/journal.pone.0026161

531 Yaffe, E., \& Tanay, A. (2011). Probabilistic modeling of Hi-C contact maps eliminates systematic

532 biases to characterize global chromosomal architecture. Nature genetics, 43(11), 1059-65.

533 doi:10.1038/ng.947 


\section{Figure 1}

\section{Hi-C insert distribution.}

The distribution of genomic distances between $\mathrm{Hi}-\mathrm{C}$ read pairs is shown for read pairs mapping to each chromosome. For each read pair the minimum path length on the circular chromosome was calculated and read pairs separated by less than 1000 bp were discarded. The 2.5 Mb range was divided into 100 bins of equal size and the number of read pairs in each bin was recorded for each chromosome. Bin values for each chromosome were normalized to sum to 1 and plotted.

\section{$\mathrm{Hi}-\mathrm{C}$ read pair insert distributions}

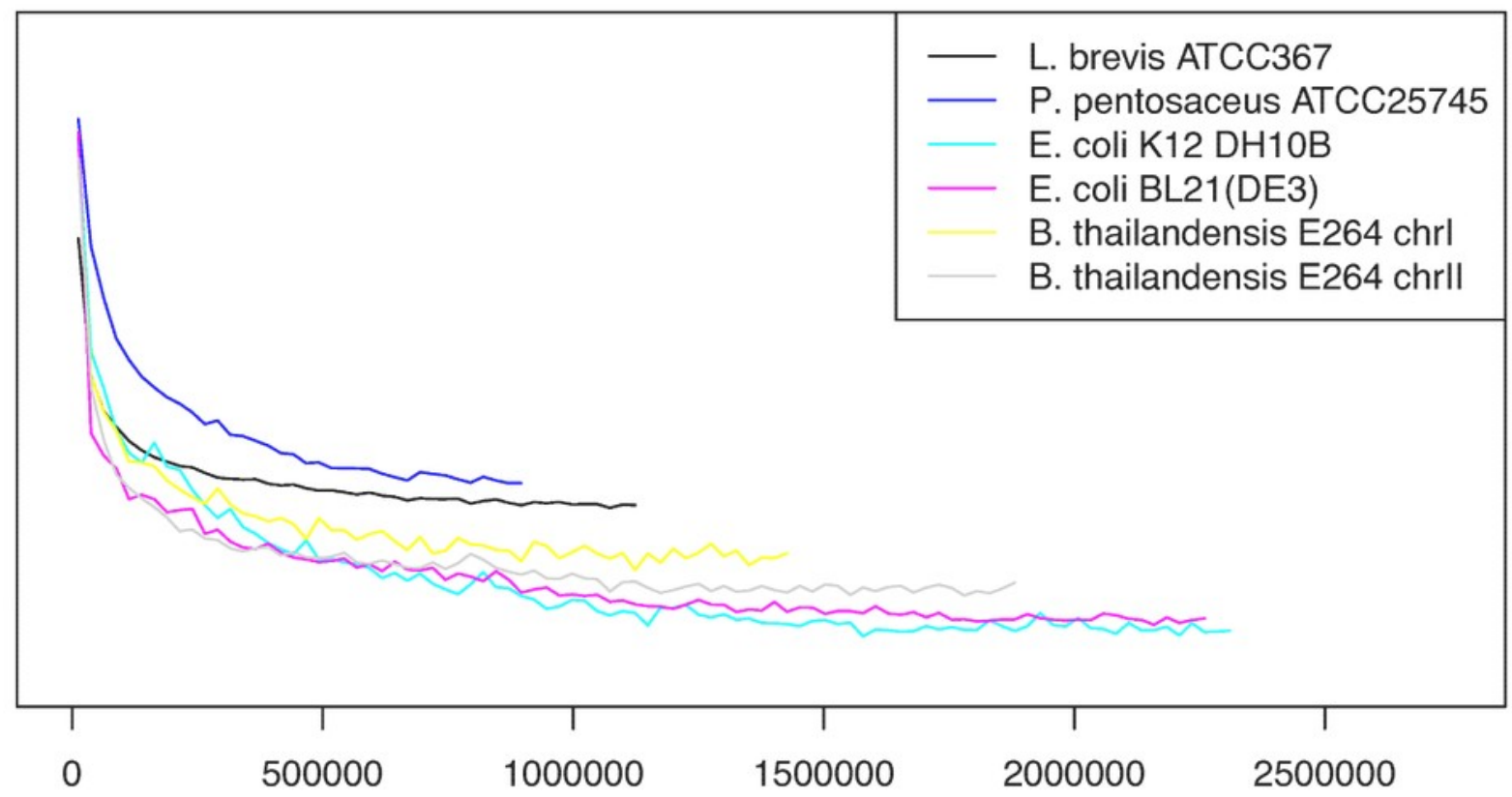

Insert distance in nucleotides 


\section{Figure 2}

\section{Metagenomic Hi-C associations.}

The log-scaled, normalized number of $\mathrm{Hi}-\mathrm{C}$ read pairs associating each genomic replicon in the synthetic community is shown as a heat map (see color scale, blue to yellow: low to high normalized, log scaled association rates). Bur1: $B$. thailandensis chromosome 1. Bur2: $B$. thailandensis chromosome 2. Lac0: L. brevis chromosome, Lac1: L. brevis plasmid 1, Lac2: L. brevis plasmid 2, Ped: P. pentosaceus, K12: E. coli K12 DH10B, BL21: E. coli BL21.

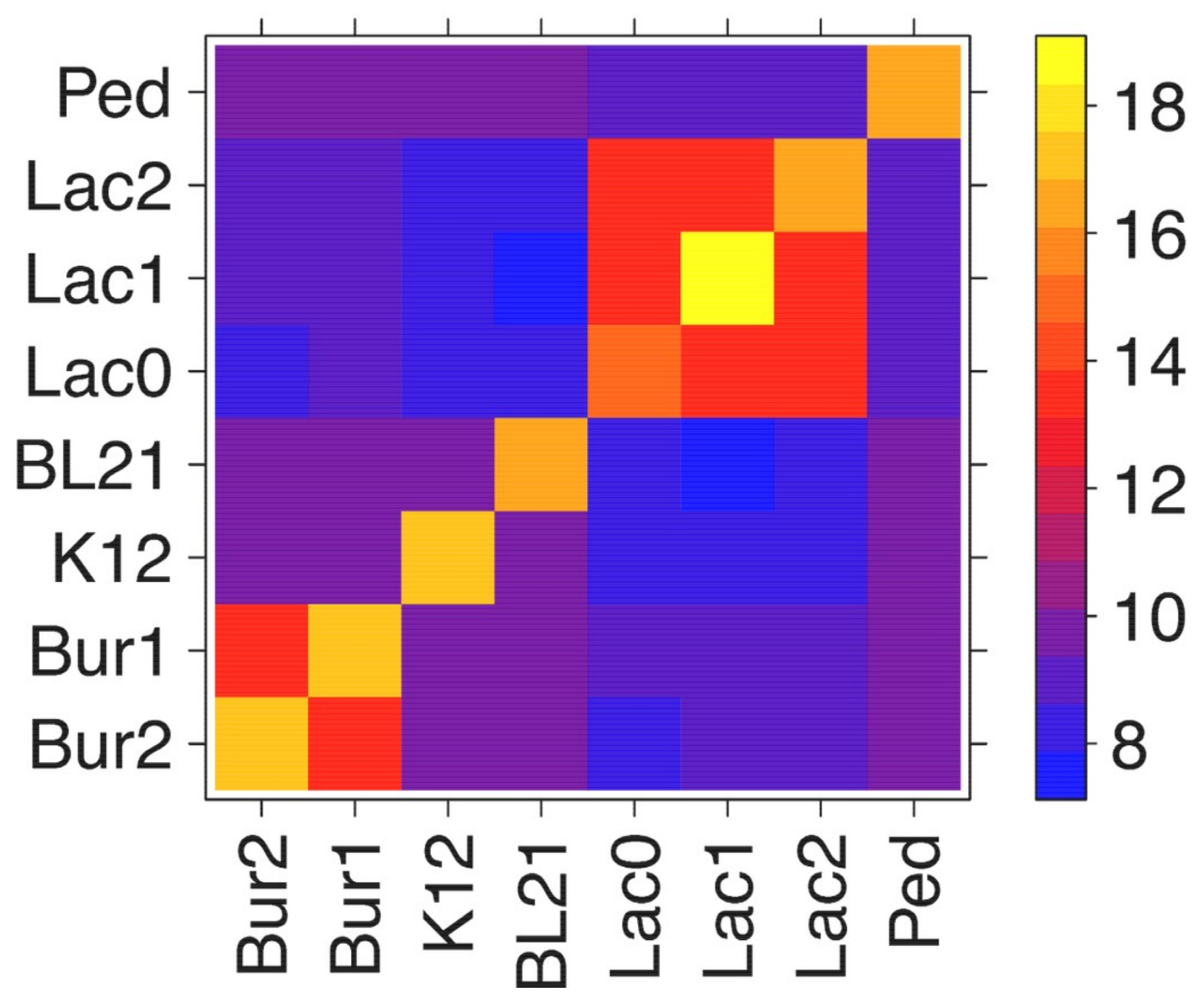




\section{Figure 3}

\section{Contigs associated by $\mathrm{Hi}-\mathrm{C}$ reads.}

A graph is drawn with nodes depicting contigs and edges depicting associations between contigs as indicated by aligned $\mathrm{Hi}-\mathrm{C}$ read pairs, with the count thereof depicted by the weight of edges. Nodes are colored to reflect the species to which they belong (see legend) with node size reflecting contig size. Contigs below $5 \mathrm{~kb}$ and edges with weights less than 5 were excluded. Contig associations were normalized for variation in contig size.

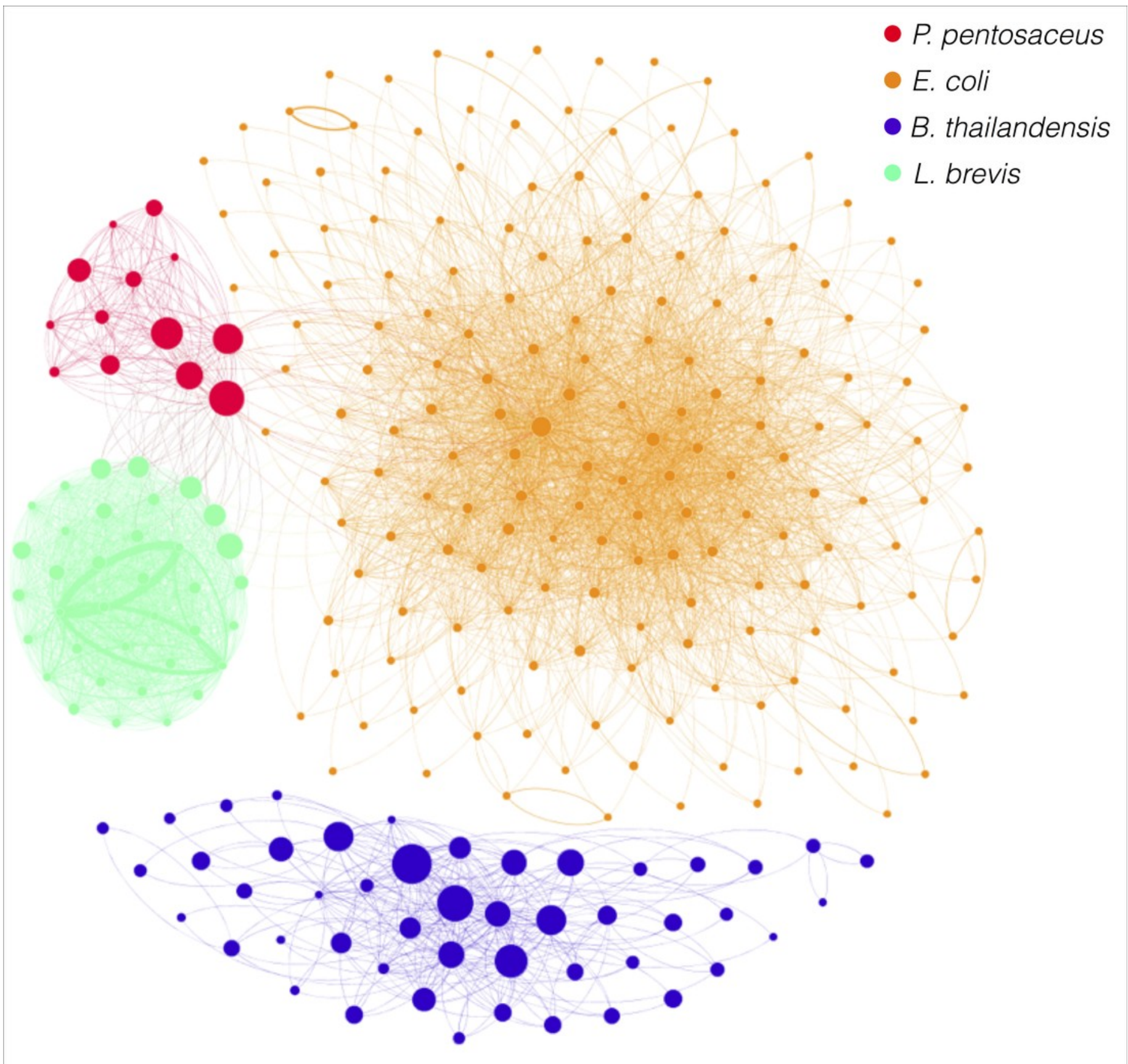




\section{Figure 4}

\section{Hi-C contact maps for replicons of Lactobacillus brevis.}

Contact maps show the number of $\mathrm{Hi}-\mathrm{C}$ read pairs associating each region of the $\mathrm{L}$. brevis genome. The L. brevis chromosome (LacO, a, Spearman rank correlation) and plasmids (Lac1, b; Lac2, c) show enrichment for local associations (bright diagonal band). Interactions between Lac1 and Lac0 (d) and Lac2 and Lac0 (e) are shown. All except Lac0 are logscaled. Circularity of Lac0 became apparent after transforming data with the Spearman rank correlation (computed for each matrix element between the row and column sharing that element) in place of log transformation (a) indicated by the high number of contacts between the ends of the sequence. In all plots, pixels are sized to represent interactions between blocks sized at $1 \%$ of the interacting genomes. The number of Hindlll restriction sites in each region of sequence is shown as a histogram on the left and top of each panel.

(a)

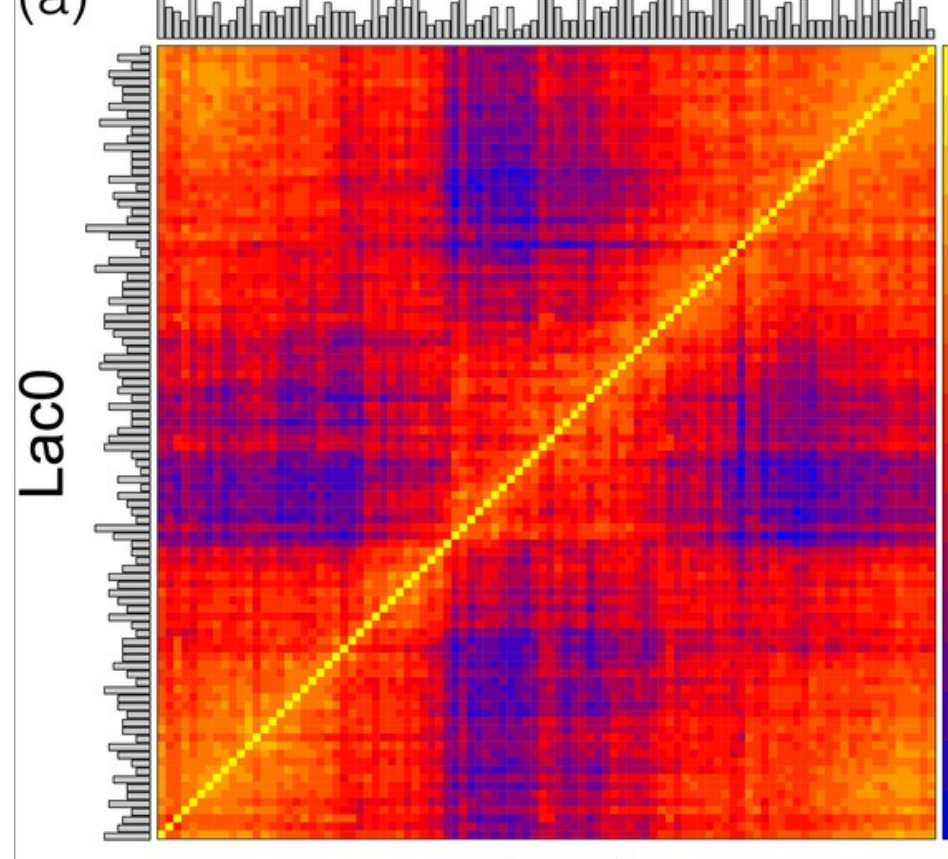

Lac0 (b)
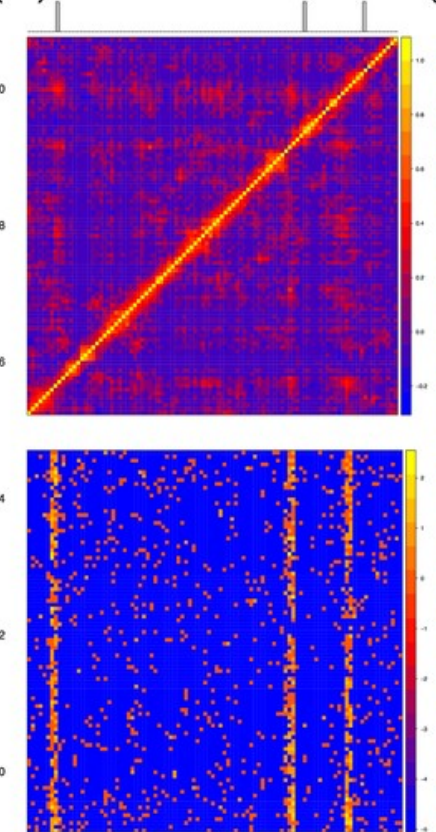

(d)
Lac1 (c)
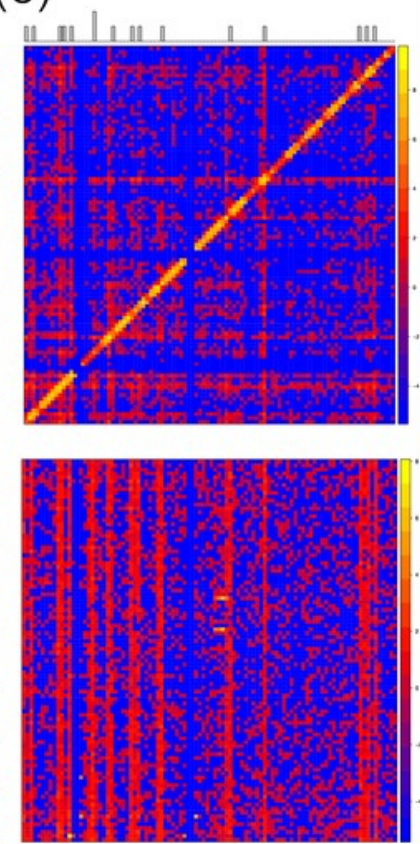

(e) 


\section{Figure 5}

Relationship of distance to degree of separation in $\mathrm{Hi}-\mathrm{C}$ and mate-pair variant graphs.

The length of paths between random pairs of SNP sites in a SNP graph constructed from both $\mathrm{Hi}-\mathrm{C}$ and mate-pair libraries of varying sizes (left; $5 \mathrm{~kb}, 10 \mathrm{~kb}, 20 \mathrm{~kb}, 40 \mathrm{~kb}$ ), smoothed using locally-weighted regression.

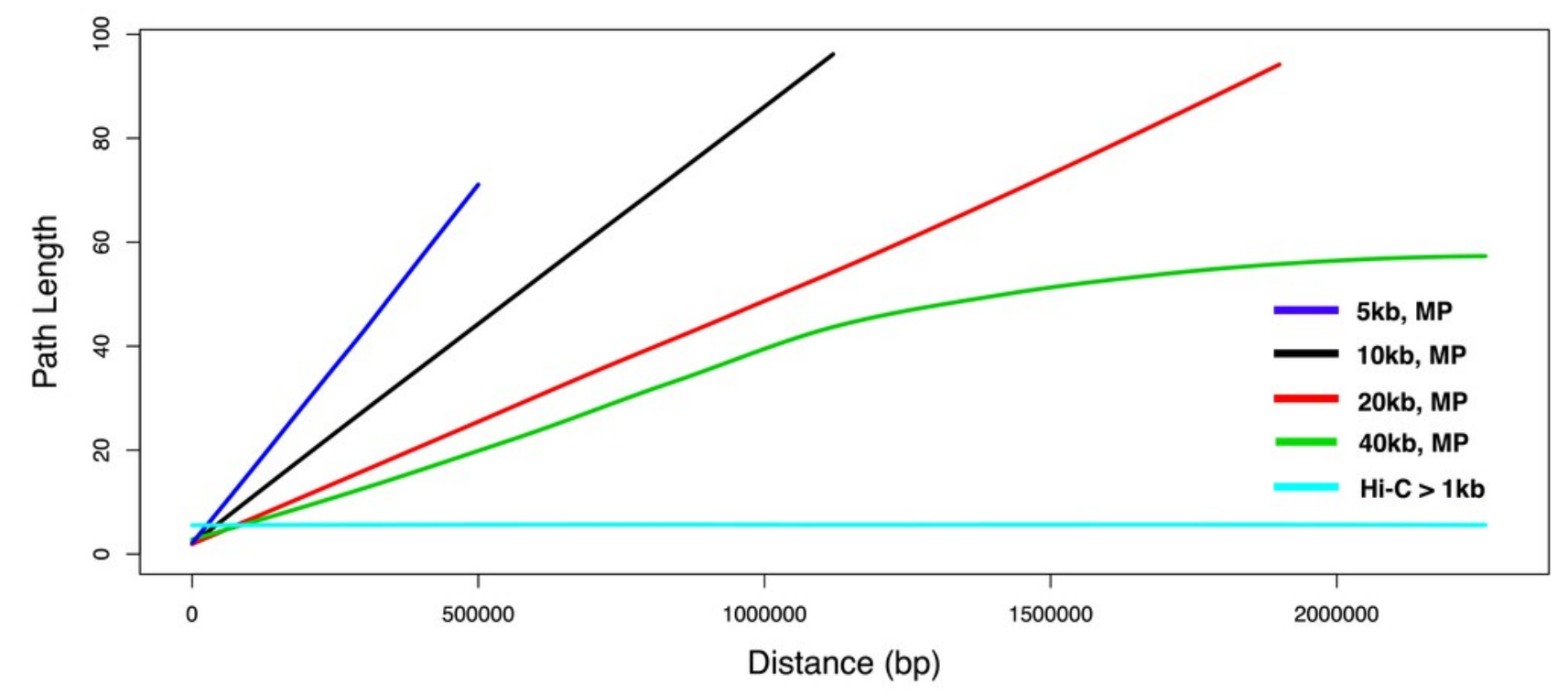




\section{Table 1 (on next page)}

\section{Species alignment fractions.}

The number of reads aligning to each replicon present in the synthetic microbial community are shown before and after filtering, along with the percent of total constituted by each species. The GC content ("GC") and restriction site counts (“\#R.S.") of each replicon, species, and strain are shown. Bur1: $B$. thailandensis chromosome 1. Bur2: B. thailandensis chromosome 2. Lac0: L. brevis chromosome, Lac1: L. brevis plasmid 1, Lac2: L. brevis plasmid 2, Ped: P. pentosaceus, K12: E. coli K12 DH10B, BL21: E. coli BL21. An expanded version of this table can be found in Table S2. 


$\begin{array}{lrrrrrrr}\text { Sequence } & \text { Alignment } & \text { \% of Total } & \text { Filtered } & \text { \% of Aligned } & \text { Length } & \text { GC } & \text { \#R.S. } \\ \text { Lac0 } & 10603204 & 26.17 \% & 10269562 & 96.85 \% & 2291220 & 0.462 & 629 \\ \text { Lac1 } & 145718 & 0.36 \% & 145478 & 99.84 \% & 13413 & 0.386 & 3 \\ \text { Lac2 } & 691723 & 1.71 \% & 665825 & 96.26 \% & 35595 & 0.385 & 16 \\ \text { Lac } & 11440645 & 28.23 \% & 11080865 & 96.86 \% & 2340228 & 0.46 & 648 \\ \text { Ped } & 2084595 & 5.14 \% & 2022870 & 97.04 \% & 1832387 & 0.373 & 863 \\ \text { BL21 } & 12882177 & 31.79 \% & 2676458 & 20.78 \% & 4558953 & 0.508 & 508 \\ \text { K12 } & 9693726 & 23.92 \% & 1218281 & 12.57 \% & 4686137 & 0.507 & 568 \\ \text { E. coli } & 22575903 & 55.71 \% & 3894739 & 17.25 \% & 9245090 & 0.51 & 1076 \\ \text { Bur1 } & 1886054 & 4.65 \% & 1797745 & 95.32 \% & 2914771 & 0.68 & 144 \\ \text { Bur2 } & 2536569 & 6.26 \% & 2464534 & 97.16 \% & 3809201 & 0.672 & 225 \\ \text { Bur } & 4422623 & 10.91 \% & 4262279 & 96.37 \% & 6723972 & 0.68 & 369\end{array}$




\section{Table 2 (on next page)}

Markov clustering of metagenome assembly contigs using Hi-C data.

A range of inflation parameters were applied, and the precision and recall for the resulting clusters was calculated as described in the text. An inflation parameter of 1.1 produced a near perfect clustering of contigs by species. 


$\begin{array}{llll}\text { Inflation } & \text { Precision } & \text { Recall } & \text { \# clusters } \\ 2.0 & 1 & 0.19 & 33 \\ 1.3 & 1 & 0.33 & 25 \\ 1.125 & 1 & 0.98 & 5 \\ 1.1 & 0.96 & 0.98 & 4\end{array}$




\section{Table 3 (on next page)}

\section{Variant graph statistics.}

Connectivity statistics are shown for variant graphs constructed from various simulated matepair (\# kb, MP) and $\mathrm{Hi}-\mathrm{C}$ read datasets. Graph constructed from all $\mathrm{Hi}-\mathrm{C}$ data are compared to those constructed using only $\mathrm{Hi}-\mathrm{C}$ read pairs with inserts over $1 \mathrm{~kb}$. The $\mathrm{Hi}-\mathrm{C}$ variant graphs are highly connected in contrast to the mate-pair graphs that have both lower connectedness and lower rates of variants occurring in the same connected components. 


\begin{tabular}{|c|c|c|c|c|}
\hline & $\begin{array}{l}\text { Num. } \\
\text { reads }\end{array}$ & $\operatorname{Max}$ & Avg. & $\begin{array}{l}\text { \% Same } \\
\text { c.c }\end{array}$ \\
\hline $5 \mathrm{~kb}, \mathrm{MP}$ & 10287315 & 71 & 14.81 & 6.21 \\
\hline 10 kb, MP & 7681515 & 96 & 24.45 & 16.6 \\
\hline $20 \mathrm{~kb}, \mathrm{MP}$ & 4871227 & 94 & 27.58 & 32.38 \\
\hline $40 \mathrm{~kb}, \mathrm{MP}$ & 4257896 & 111 & 37.19 & 100 \\
\hline $\mathrm{Hi}-\mathrm{C}$ (all) & 16429505 & 10 & 5.11 & 97.77 \\
\hline $\mathrm{Hi}-\mathrm{C}(>1 \mathrm{~kb})$ & 111525 & 11 & 5.47 & 94.7 \\
\hline
\end{tabular}

